\title{
A Condition-Based Maintenance Model for Assets with Accelerated Deterioration due to Fault Propagation
}

\author{
Zhenglin Liang, Member IEEE, Ajith Kumar Parlikad
}

\begin{abstract}
Complex industrial assets such as power transformers are subject to accelerated deterioration when one of its constituent component malfunctions, affecting the condition of other components, which is a phenomenon called fault propagation. In this paper, we present a novel approach for optimizing condition-based maintenance policies for such assets by modelling their deterioration as a multiple dependent deterioration path process. The aim of the policy is to replace the malfunctioned component and mitigate accelerated deterioration at minimal impact to the business. The maintenance model provides guidance on determining inspection and maintenance strategies to optimize asset availability and operational cost.
\end{abstract}

Index Terms-condition based maintenance, accelerated deterioration, multiple dependent deteriorating process, unrevealed malfunction.

\section{Nomenclature}

A critical component is a component whose functionality is essential for the asset to perform its designed function.

A non-critical component is a component with an associated function that may influence the condition of critical components, but is not essential for the asset to perform its designed function.

Fault propagation is an accelerated deterioration of critical components triggered by the malfunction of non-critical components.

Minor preventive maintenance is carried out to repair or maintain malfunctioned non-critical components. It prevents further damage to other components due to fault propagation, and it moves the asset from an accelerated deterioration path to the normal-paced deterioration path.

Major preventive maintenance prevents the asset from

Manuscript received $15^{\text {th }}$ December 2013.

Zhenglin Liang is with the Cambridge University Engineering Department, UK CB3 0FS (Tel: 44-1223-766141; e-mail: zl284@cam.ac.uk).

Ajith Kumar Parlikad is with the Cambridge University Engineering Department, UK CB3 0FS (Tel: 44-1223-765606; e-mail: aknp2@cam.ac.uk). reaching the deterioration failure state, and brings both the critical components and non-critical components back to an as good as new state [1].

Corrective maintenance targets sudden Poisson failures caused by an external event. It aims to bring the asset back to the rated working condition [2].

Replacement is undertaken after an asset reaches the deterioration failure state. It changes the asset condition to an as good as new state.

\section{Notation}

$k \quad$ The number of condition states of critical components before deterioration failure.

$m \quad$ The number of different accelerated deterioration paths triggered by the malfunction of non-critical components.

$\lambda_{n i, 0}$ The constant rate of deterioration of critical components from state $i$ to state $i+1$, when non-critical components are working properly.

$\lambda_{d i, j} \quad$ The constant rate of deterioration of critical components from state $i$ to state $i+1$, when non-critical components are experiencing the $j^{\text {th }}$ type of malfunction.

$\lambda_{f i, j} \quad$ The constant rate of occurrence of the $j^{\text {th }}$ type of malfunction of non-critical components when critical components are in the $i^{\text {th }}$ state.

$F \quad$ The deterioration failure state.

$b \quad$ The threshold for major preventive maintenance activity.

$1 / \lambda_{\mathrm{F}}$ The statistical mean time between revealed Poisson failures.

$1 / \lambda_{\text {Fd }}$ The statistical mean time between revealed Poisson failures, when non-critical components are malfunctioned.

$1 / \lambda_{\text {in }}$ The statistical mean time between two successive inspections.

$1 / \mu_{\text {in }}$ The statistical mean duration of inspection.

$1 / \mu_{\mathrm{c}}$ The statistical mean duration of minor preventive maintenance.

$1 / \mu_{\mathrm{F}}$ The statistical mean duration of corrective repair for 
revealed Poisson failure.

$1 / \mu_{\mathrm{M}}$ The statistical mean duration of major preventive maintenance.

$1 / \mu_{R}$ The statistical mean duration of replacement.

$\mathrm{C}_{\text {in }}^{\prime} \quad$ The cost of each inspection.

$\mathrm{C}_{\mathrm{c}}^{\prime} \quad$ The cost of each minor preventive maintenance.

$\mathrm{C}_{\mathrm{F}}^{\prime} \quad$ The cost of each corrective maintenance after Poisson failure.

$\mathrm{C}_{\mathrm{M}}^{\prime} \quad$ The cost of each major preventive maintenance.

$\mathrm{C}_{\mathrm{R}}^{\prime} \quad$ The cost of each replacement activity.

$\mathrm{C}_{\mathrm{u}} \quad$ The unplanned per unit down time cost.

$\mathrm{C}_{\mathrm{p}} \quad$ The planned per unit down time cost.

$\pi_{i, j} \quad$ The steady state probability of state $(i, j)$.

$A_{S} \quad$ The availability of the asset.

$C_{S} \quad$ The time-averaged operating cost of the asset.

$C_{T} \quad$ The cost of downtime.

$C_{A} \quad$ The time-averaged costs of each activity.

$f(t)$ The lifetime function of the asset.

$\boldsymbol{\alpha} \quad$ The probability vector denoting the initial probability of starting in any state.

$\boldsymbol{S} \quad$ The transition matrix.

Abbreviations

CTMC Continuous time Markov chain

MDDPM Multiple dependent deterioration path model

SDPM Single deterioration path model

MTBI Mean time between inspections

\section{INTRODUCTION}

I ndustrial assets are composed of multiple components with different fault tolerances and life expectancies. In some occurrences, a non-critical component within the asset might be damaged by external events and become malfunctioned, and in turn have an adverse impact on other components, which is normally observed as a general result of an excessive decay of the asset. The excessive decay induced by the malfunctioned component could be mitigated by a minor maintenance, if it is discovered on time. However, if left unattended, it will result in failure. This phenomenon is called fault propagation. It is an event-triggered latent process with a prolonged impact on the asset aging rate. In practice, the phenomenon of fault propagation can be identified by assessment methods such as fault tree analysis [3], and failure mode and effect analysis [4]. Under this situation, modelling the asset aging process as one indistinguishable deterioration path seems to be oversimplified, and unlikely to withstand empirical scrutiny. This condition demands a refinement of asset deterioration modelling, and reforming of the condition-based maintenance strategy, to further improve asset performance by timely observation and mitigation of the accelerated aging associated with fault propagation.

The reliability engineering community has been battling with the difficulty of modeling the complex behavior of asset deterioration and optimizing its maintenance. Currently, one of the key research areas receiving increasing attention is to use mathematical models to capture the phenomenon of dependencies among components within an asset (or among assets within a system). Amongst these dependencies, stochastic dependence, defined by Dekker [5] as where "...the state of a component influences the lifetime distribution of other components, or there are causes outside the system which bring about simultaneous failures (so-called common cause failures)" is most challenging and underdeveloped (and hence the least progressed).

Stochastic dependence is manifested in two ways in a multi-component asset: inherent dependence, and induced dependence. Inherent dependence is where the deteriorations of components in an asset are affected by one another throughout their lifetimes. On the other hand, induced dependence is where a malfunction in one component of an asset will affect the rate of deterioration of another component in that asset. We will now discuss the current literature on inherent dependence.

A multivariate competing risk model is intuitively appropriate for modelling this type of dependence. Gorden [6] developed a generalized dependent risk model where the effect of risks is drawn randomly from a multivariate normal distribution, whose covariate matrix expresses the pairwise correlation between risks. Copulas are an attractive alternative to model inherent dependence statistically, as it is a function of joint probability, by linking univariate marginal distributions. Lo and Wike [7] implemented an Archimedean copula to model multiple dependent competing risks. Wang and Pham [8] formulated the inherent dependence among multiple degrading processes by selecting the best fitting copulas as well as the time varying copulas. Tang [9] argued that copulas might not be uniquely determined under incomplete information. Moreover, the frequently implemented Gaussian copulas may induce gross error on calculated system reliability. Bain [10] designed a novel deterioration rate interaction approach where he modelled the effect of degradation state of one component on the degradation rate of other components in the system. We have discussed a number of approaches found in literature to model inherent dependence. All these approaches have a common factor: they use a multi-variate distribution or Copulas to represent the joint ageing process of the components.

As opposed to inherent dependence which could occur in the components in an asset throughout its life, induced dependence manifests after an internal or external trigger event. For instance, an abnormal surge of current could cause a clamping in a transformer to become loose due to electromagnetic forces (malfunction), which can increase vibrations in the transformer, thereby accelerating the deterioration of certain other components. Failure interaction models are one of the main streams to analytically describe the interactive effect induced 
after an internal event, for instance, a component failure. There are abundant articles in this area. Nicolai and Dekker [11] refined a three-type classification scheme, which was originally introduced by Murthy and Nguyen [12] into a well-utilized two-type classification. Type I assumes that the failure of one component may instantaneously cause the failure of another component with a certain probability. Scarf and Deara [13] modeled the type I failure dependence in a two-component system, and employed failure-based replacement, age-based replacement, and opportunistic age-based replacement, considering set up cost. Type II models the nonlethal effect after the failure of one component, which can be further subdivided into two categories: failure interaction, and shock damage interaction. Failure interaction models the influence of failure of one component on the hazard rate or hazard function of other components. Lai [14] demonstrated failure interaction in a two-unit parallel system, where the failure of unit 1 will increase the instantaneous failure rate of unit 2 , and the failure of unit 2 will cause instantaneous failure of unit 1. A replacement policy based of the number of failures on unit 1 is optimized to minimize the time averaged operating cost of the system. Golmaknmi [15] modeled the failure interaction in a two-component repairable system, where the failure of unit 1 is unrevealed and increases the system operation cost, yet the failure of unit 2 will cause the system to cease operating and also increase the instantaneous failure rate of unit 1 . The paper minimized the systemic operation cost in a finite time horizon by varying the inspection interval on unit 1 . Shock damaged interaction considers the case where the failure of one component will induce a shock damage on other components, and this damage may accumulate over time. Satow [16] constructed a model for a two-component system with shock-damaged interaction, where the non-homogeneous failure of unit 1 will induce a random amount of damage on unit 2 , and eventually result in high cost system failure. To prevent the cumulative damage on unit 2 from exceeding a predefined threshold, a preventive replacement policy based on both system age and cumulative damage to unit 2 are implemented to minimize the time-averaged operating cost. Wang [17] model the same shock interaction model on two-component systems, but in his case unit 2 is repairable with a geometric process.

Induced dependence may also be triggered by external risk. Common cause failure [18], [19] is a well-known body of research, which belongs to this area. Common cause failure plays an important role in the estimation of system reliability. Kavm [20] found that the assumption of independent failures leads to the system reliability being significantly overestimated, so he proposed a Bayesian model to infer the common cause failure probability based on historical convoluted data. Xing [21] considered a special type of common cause failure: propagated failure with a global effect on a binary state system. She modeled a logic framework using a dynamic fault tree, additionally introducing the functional dependence gate to represent the competition in the time domain between failure isolation and propagated failure. Levitin [22] evaluated the reliability of a serial-parallel multi-state system subject to common cause failure by employing a universal moment generating function, which is more tolerant of changes to system topology than fault tree analysis. Further developments are made in his later publications on modeling the selective effect of propagated failure [23], and randomizing the propagation time of failure [24]. The models about induced dependence normally explicate its trigger event and analytically formulate its sub-sequential effects. In other words, it highlights the changes in system aging characteristics before and after the trigger event.

The phenomena of both inherent co-degradation and induced failure interaction are well captured and modeled in current literature. In this paper, we attempt to draw the attention of researchers and practitioners to a phenomenon caused by an internal stochastic dependence among components, which manifests a different type of characteristic: fault propagation. Fault propagation is an induced co-degradation, which is triggered by an unrevealed event such as a malfunction of components or an induced agent of degradation. In practice, the phenomenon of fault propagation has been widely observed in industrial assets with complex structures. For instance, in power transformers, if the bush gasket becomes inelastic, the ingress moisture and oxygen in insulation oil will catalyse the hydrolysis and oxidation of the insulation paper, and reduce the lifetime of the power transformer. As reported in Shroff and Stannet [25], under $2 \%$ moisture, the lifetime of a power transformer can be reduced from the original 38 years to 1.9 years.

In this paper, we focus on modelling the effect of fault propagation within an asset, where the co-degradation among components could be seen as the acceleration of the internal clock of asset deterioration. The induced co-degradation phenomenon is therefore modelled as a transition from normal asset deterioration to the resulting accelerated deterioration. By modelling it this way, the deterioration model does not require detailed component level data (which is hard to obtain in practice), it only requires data at the asset level.

In particular, we model the asset deterioration behaviour using a multiple dependent deterioration path model (MDDPM) constructed using a continuous time Markov chain (CTMC). There are a number of papers [26]-[33] that use CTMC to model the deterioration and maintenance of a multi-state single unit asset and find closed form solutions for availability and cost. In addition, a number of papers (e.g., [34], [35]) present models for multi-directional degradation using Markov decision processes. Amari suggested in [36] that ideally the different deterioration mechanisms in an asset should each be modelled. Further, he suggests that the model should reflect that certain maintenance activities can reduce the deterioration rate of the asset. In our modeling approach, we model the normal deterioration and accelerated deterioration of the asset, and highlight the transition between them. Such a transition may happen at any state of deterioration. 
We demonstrate the significance of modeling the accelerated deterioration associated with fault propagation in Section II. In Section III, we design a condition-based maintenance model which generates a periodic inspection plan to detect fault propagation. In addition, the model also provides a maintenance strategy to alleviate the detrimental effect of accelerated deterioration and restore the system back to normal-paced ageing. Section VI uses a case example from the power industry to bring the mathematical models to life. Section V summarizes the findings, and recommends the direction of future work.

\section{DETERIORATION MODEL}

The deterioration model we put forward is for a multi-component asset, which is composed of critical and non-critical components. We formulate the deterioration model as a MDDPM. A comparison is made between a single deterioration path model (SDPM) and a MDDPM. The result shows the accelerated deterioration associated with fault propagation has a non-negligible impact on the asset lifetime function, which is also known as the unreliability function associated with asset aging. This result also services as a rationale for modeling the asset deterioration as a MDDPM.

\section{A. Deterioration model formulation}

We assume that the asset deterioration is a Markovian process. The state of the asset is defined by the condition of both critical and non-critical components, and the sojourn time at each state is exponentially distributed. Further, the joint probability of the simultaneous occurrence of the state transition of critical components and the malfunctioning of a non-critical component is assumed to be negligible. We implement a terminating Markov chain to construct the MDDPM as shown in Fig. 1.

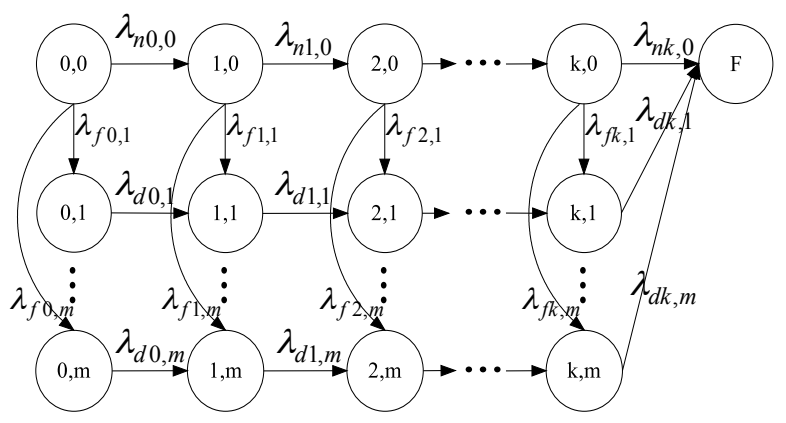

Fig. 1. State diagram of asset deterioration model.

The asset states in the model are characterized by two indices $i$ and $j$, where $i$ signifies the condition of critical components, and $j$ denotes the condition of non-critical components. $i=0$ implies that critical components are new, and will monotonically decay to the failure state $(F)$. Under normal use conditions, the critical components are assumed to deteriorate at the constant hazard rate $\lambda_{n i, 0}$. However, if a malfunction $(1 \leq j \leq m)$ happens on non-critical components, the constant deterioration rate of critical components will accelerate to $\lambda_{d i, j}$. The different $j$ index indicates different malfunction modes of non-critical components. The rate at which the $j^{\text {th }}$ type of malfunction happens when the critical components are at the $i^{\text {th }}$ state is denoted by $\lambda_{f i, j}$.

\section{B. Estimated lifetime function}

We model the lifetime distribution of an asset as a multi-variate function of multiple constant deterioration rates and transition rates between them. By recalling the state diagram in Fig. 1, we can see that the lifetime of an asset is identical to the time for the asset to reach the absorbing state $F$. Thus, in the model, the lifetime distribution of the asset could be modeled by a phase-type distribution [37], [38].

We will now demonstrate the method of calculating the lifetime distribution using a simplified case. Consider an asset that deteriorates homogeneously $\left(\lambda_{n 0,0}=\lambda_{n 1,0}=\cdots \lambda_{n k, 0}=\lambda_{n}\right.$, and $\lambda_{d 0, j}=\lambda_{d 1, j}=\cdots=\lambda_{d k, j}=\lambda_{d}$ ). Also consider that the asset has two deterioration states, and one accelerated deterioration path (see Fig. 2). The malfunction rate of non-critical components is invariant with the deterioration condition of critical components $\left(\lambda_{f 0, j}=\lambda_{f 1, j}=\cdots=\lambda_{f k, j}=\right.$ $\left.\lambda_{f}\right)$.

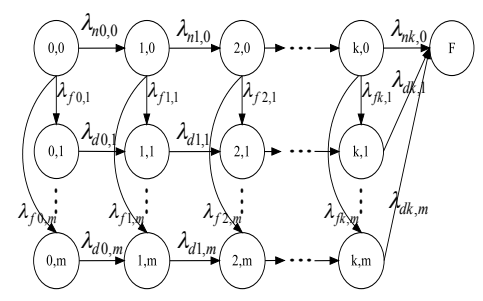

Fig. 2. Homogeneous deterioration process.

We can calculate the lifetime distribution of this asset using a phase type distribution as

$f(t)=\boldsymbol{\alpha} e^{\mathrm{St}} \mathbf{S}^{0}$.

The parameter setting for this case is

$$
\begin{gathered}
\mathbf{S}=\left[\begin{array}{cccc}
-\left(\lambda_{n}+\lambda_{f}\right) & \lambda_{f} & \lambda_{n} & 0 \\
0 & -\lambda_{d} & 0 & \lambda_{d} \\
0 & 0 & -\left(\lambda_{n}+\lambda_{f}\right) & \lambda_{f} \\
0 & 0 & 0 & -\lambda_{d}
\end{array}\right], \\
\alpha=\left[\begin{array}{cccc}
1 & 0 & 0 & 0
\end{array}\right],
\end{gathered}
$$

and

$$
\mathbf{S}^{0}=-\mathbf{S} \mathbf{1}=\left[\begin{array}{c}
0 \\
0 \\
\lambda_{n} \\
\lambda_{d}
\end{array}\right],
$$

where 1 is a 4 x 1 vector with every element being 1 .

As $\mathbf{S}$ is an upper triangular matrix, it has two double roots which are $\lambda_{1}=-\lambda_{d}$, and $\lambda_{2}=-\left(\lambda_{n}+\lambda_{f}\right)$. We can calculate the eigenvectors and generalized eigenvectors of matrix $\mathbf{S}$.

$\left(\mathbf{S}-\lambda_{1} \mathbf{I}\right) \mathbf{V}_{1}=0$,

$\mathbf{V}_{1}=\left[\begin{array}{llll}1 & \frac{\lambda_{n}+\lambda_{f}-\lambda_{d}}{\lambda_{f}} & 0 & 0\end{array}\right]$, 
$\left(\mathbf{S}-\lambda_{1} \mathbf{I}\right) \mathbf{V}_{2}=\mathbf{V}_{1}$,

$\mathbf{V}_{2}=\left[\begin{array}{llll}1 & \frac{\lambda_{d}+\lambda_{d} \lambda_{n}+\lambda_{d} \lambda_{f}-\lambda_{d}^{2}-\lambda_{n}}{\lambda_{d} \lambda_{f}} & \frac{1}{\lambda_{d}} & \frac{\lambda_{n}+\lambda_{f}-\lambda_{d}}{\lambda_{f}}\end{array}\right]$,

$\left(\mathbf{S}-\lambda_{2} \mathbf{I}\right) \mathbf{V}_{3}=0$,

$\mathbf{V}_{3}=\left[\begin{array}{llll}1 & 0 & 0 & 0\end{array}\right]$,

$\left(\mathbf{S}-\lambda_{2} \mathbf{I}\right) \mathbf{V}_{4}=\mathbf{V}_{3}$,

$\mathbf{V}_{4}=\left[\begin{array}{llll}1 & 0 & 1 / \lambda_{n} & 0\end{array}\right]$.

We combine the four eigenvectors and generalized eigenvectors to a $4 \times 4$ matrix $\mathbf{P}=\left[\begin{array}{llll}\mathbf{V}_{1}^{T} & \mathbf{V}_{2}^{T} & \mathbf{V}_{3}^{T} & \mathbf{V}_{4}^{T}\end{array}\right]$. This combination enables us to further decompose the matrix $\mathbf{S}$ as follows.

$\mathbf{S}=\mathbf{P J P}^{-1}$

where

$\mathbf{J}=\left[\begin{array}{cccc}-\lambda_{d} & 1 & 0 & 0 \\ 0 & -\lambda_{d} & 0 & 0 \\ 0 & 0 & -\left(\lambda_{n}+\lambda_{f}\right) & 1 \\ 0 & 0 & 0 & -\left(\lambda_{n}+\lambda_{f}\right)\end{array}\right]$.

$f(t)$ can therefore be rewritten as

$f(t)=\boldsymbol{\alpha} \mathbf{P} e^{I t} \mathbf{P}^{-1} \mathbf{S}^{0}$,

$e^{\mathrm{J} t}=\left[\begin{array}{cccc}e^{-\lambda_{d} t} & t e^{-\lambda_{d} t} & 0 & 0 \\ 0 & e^{-\lambda_{d} t} & 0 & 0 \\ 0 & 0 & e^{-\left(\lambda_{n}+\lambda_{f}\right) t} & t e^{-\left(\lambda_{n}+\lambda_{f}\right) t} \\ 0 & 0 & 0 & e^{-\left(\lambda_{n}+\lambda_{f}\right) t}\end{array}\right]$.

The solution of $f(t)$ for this model is

$f(t)=\frac{\lambda_{d} \lambda_{n} \lambda_{f}^{2}}{\left(\lambda_{n}+\lambda_{f}-\lambda_{d}\right)^{2}}\left[-\frac{\left(\lambda_{d}-\lambda_{n}\right) e^{-\lambda_{d} t}}{\lambda_{n} \lambda_{f}}+\right.$

$\frac{\left(\lambda_{d} \lambda_{n}+\lambda_{d} \lambda_{f}-\lambda_{d}^{2}\right) t e^{-\lambda_{d} t}}{\lambda_{n} \lambda_{f}}+\frac{\left(\lambda_{d}-\lambda_{n}\right) e^{-\left(\lambda_{n}+\lambda_{f}\right) t}}{\lambda_{n} \lambda_{f}}+$

$\left.\frac{\lambda_{n}\left(\lambda_{n}+\lambda_{f}-\lambda_{d}\right)^{2} t e^{-\left(\lambda_{n}+\lambda_{f}\right) t}}{\lambda_{d} \lambda_{f}^{2}}-\frac{\left(\lambda_{n}+\lambda_{f}-\lambda_{d}\right) t e^{-\left(\lambda_{n}+\lambda_{f}\right) t}}{\lambda_{f}}\right]$.

To verify the above result, when the unrevealed malfunction rate $\lambda_{f}$ is $0, f(t)$ reduces to $\lambda_{n}^{2} t e^{-\lambda_{n} t}$ which is identical to that of a single deteriorating path model SDPM with an averaged sojourn time at each transient state equal to $1 / \lambda_{n}$ [39].

\section{Comparison between SDPM and MDDPM}

It is intuitive that the difference of the average lifetimes between the SDPM and MDDPM depends on two rates $l_{1}$, and $l_{2}$, where $l_{1}=\lambda_{f} / \lambda_{n}$, and $l_{2}=\lambda_{d} / \lambda_{n}$. The normalised difference $(e)$ can be expressed as

$e=\left|\frac{t_{I}-t_{d}}{t_{I}}\right|$

where $t_{I}$ is the average lifetime as calculated by the single deterioration process model, and $t_{d}$ is the averaged lifetime as calculated by our model. $t_{I}$, and $t_{d}$ can be calculated as

$t_{I}=\int_{0}^{\infty} \lambda_{n}^{2} t^{2} e^{-\lambda_{n} t} \mathrm{dt}$,

$t_{d}=\int_{0}^{\infty} t f(t) \mathrm{dt}$.

In Fig. 3 , we plot $e$ where $l_{1}$, and $l_{2}$ vary from 0.05 to 0.5 , and 0 to 10 respectively. For the given set of input values, note that the difference between the estimated averaged lifetimes between the two models can be as much as $40 \%$.

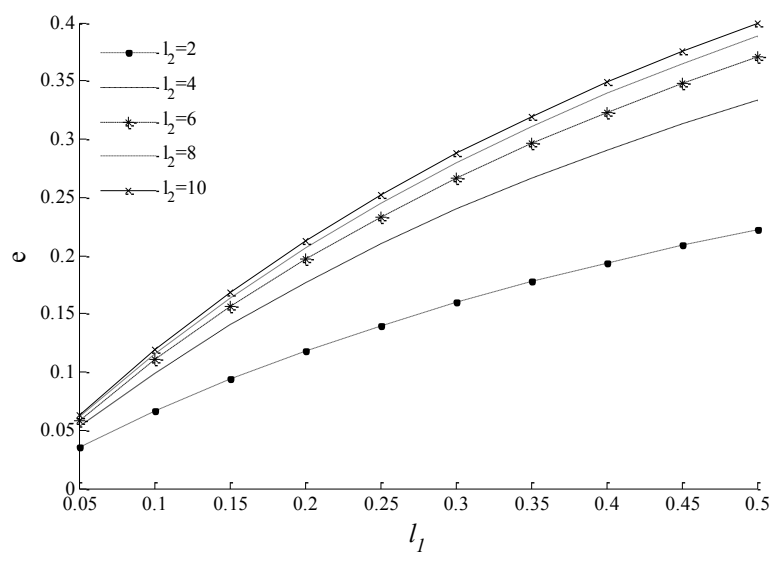

Fig. 3. Difference between estimated assets' averaged lifetimes.

The induced accelerated deterioration associated with fault propagation has a non-negligible impact on asset life. However, implementing a maintenance strategy designed to replace the malfunctioned components and alleviate resulting accelerated deterioration in a timely manner can mitigate this risk.

\section{MAINTENANCE MODEL}

In this section, we aim to build a condition based maintenance model upon the MDDPM to timely inspect and rectify the accelerated deterioration. In addition, by varying the decision variables of the $s$-mean time between inspections (MTBI) and the major preventive maintenance threshold, we are able to optimize the asset's performance in term of availability and cost. The detail of the condition based maintenance strategy is explained with detail in Subsection $C$, and the generalized state transition diagram is illustrated in Fig. 4.

\section{A. Model formulation}

In the model, states with $j=m+1$ represent inspection without further action. $j=m+2$ indicates inspection that will lead to a maintenance action. $j=m+3$ represents the maintenance action; and $j=m+4$ represents the Poisson failure state. The deterioration states $(0 \leq i \leq k, 0 \leq j \leq m)$ of the asset are unrevealed, but could be observed and assessed by periodical inspection. The Poisson failure $(j=m+4)$ and deterioration failure $(F)$ are self-announcing. The states of the asset, except for the failure state, are only revealed upon inspection. We assume that the duration for which the asset stays at a particular state is exponentially distributed. When the asset degrades to the deterioration failure state $F$, it is overhauled or replaced to restore the asset to an as good as new 
state. Inspection is performed to assess the condition of critical and non-critical components. If the non-critical components are operating in the rated condition $(j=0)$, and the critical components are in an acceptable condition $(i \leq b)$, no maintenance action is required. If the critical components are already in a bad state $(i>b)$, major preventive maintenance will be performed to bring the asset back to an as good as new state. However, even if the critical components are in a good state, if the non-critical components are subject to malfunction ( $i \leq b, j \in\{1, \cdots, m\}$ ), to prevent the accelerated deterioration associated with fault propagation, minor preventive maintenance of the malfunctioned non-critical component is required. If critical components are already degraded to a bad condition through accelerated deterioration, major preventive maintenance will be performed to bring the asset back to an as good as new condition. Apart from deterioration failure, Poisson failure with a constant hazard function $\lambda_{F}$ may occur at any deterioration state, and this condition will lead the asset to stop functioning.

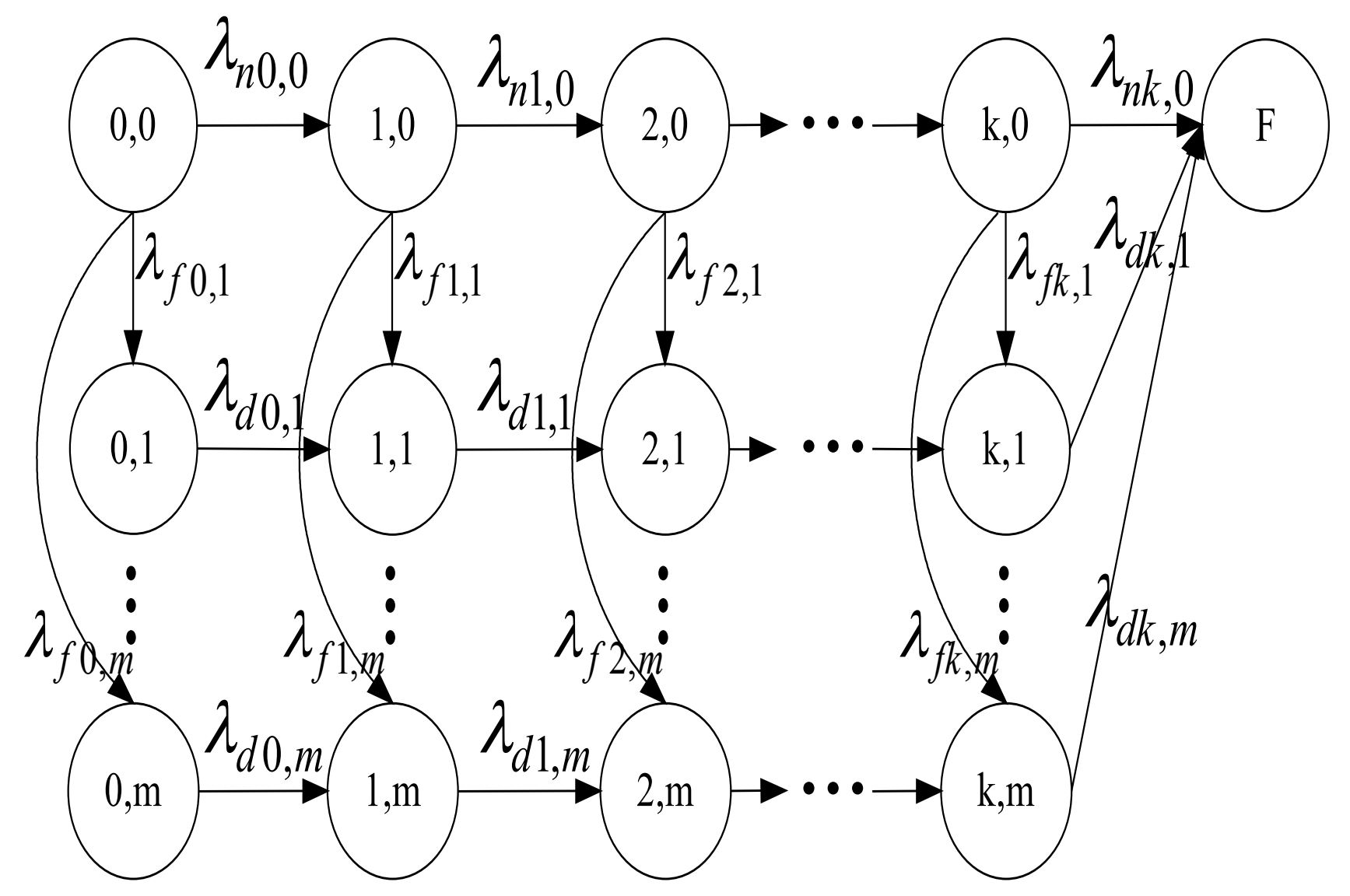

Fig. 4. Generalized continuous time Markov chain maintenance model.

When the asset is undergoing accelerated deterioration, the resistance to exogenous harmful events will be reduced, and the asset will become more vulnerable. Hence we use $\lambda_{F D}$ to indicate the higher probability of triggering a Poisson failure given that the asset is already in the accelerated deterioration process. This approach is similar to the faulty trigger failure process as mentioned by Kuo and Zou [40]. A corrective maintenance will then be performed to fix the failed component and bring the asset back to the working state just before the failure state.

\section{B. General solution}

Based on the designed CTMC model, the availability of an asset can be expressed in terms of the steady state probabilities as shown in (15).

$$
A_{S}=\sum_{i=0}^{k} \sum_{j=0}^{m} \pi_{i, j}
$$

The time-averaged operating cost contains two parts, which are the cost of downtime $C_{T}$, and the time-averaged costs of each activity $C_{A}$ (inspection and maintenance).

$C_{S}=C_{T}+C_{A}$

The cost of downtime is subdivided into two classes: planned downtime cost, and unplanned downtime cost. The planned cost is caused by the idle time of the scheduled inspection and maintenance. The unplanned down time is induced by events of sudden failure, and deterioration failure. The downtime cost is 
calculated by multiplying the per unit downtime cost with the relevant steady state probabilities as in (17).

$$
\begin{gathered}
C_{T}=C_{p}\left(\sum_{i=0}^{b} \pi_{i, m+1}+\sum_{i=0}^{k} \pi_{i, m+2}+\sum_{i=0}^{k} \pi_{i, m+3}\right) \\
+C_{u}\left(\sum_{i=0}^{k} \pi_{i, m+4}+\pi_{F}\right)
\end{gathered}
$$

The time averaged cost of each activity considers the cost of each inspection, minor preventive maintenance, major preventive maintenance, corrective maintenance, and replacement. The way to calculate this cost is to divide the cost of each activity by the duration of the activity multiplied by the relevant steady state probabilities as shown in (18).

$$
\begin{aligned}
C_{A}=C_{i n}^{\prime}\left(\sum_{i=0}^{b} \mu_{i n}\right. & \left.\pi_{i, m+2}+\sum_{i=0}^{b} \mu_{i n} \pi_{i, m+1}\right) \\
& +C_{c}^{\prime}\left(\sum_{i=0}^{b} \mu_{c} \pi_{i, m+3}\right) \\
& +C_{M}^{\prime}\left(\sum_{i=0}^{k} \mu_{M} \pi_{i, m+3}\right) \\
& +C_{F}^{\prime}\left(\sum_{i=0}^{k} \mu_{F} \pi_{i, m+4}\right)+C_{R}^{\prime} \mu_{R} \pi_{F}
\end{aligned}
$$

To calculate the availability and operational cost of the asset, we need to know the steady state probabilities. However, the model contains $(m+4)(k+1)+b+2$ interconnected states, and thus finding analytical expressions for them is complicated.

Fortunately, only the deterioration states $(j \leq m)$ are inter-state dependent. The inspection states $(m+1 \leq j \leq m+2)$ and maintenance states $(m+3 \leq j \leq m+4)$ are only related to the deterioration states in the current path. Furthermore, when $1 \leq i$ $\leq b$, the deterioration states follow a certain pattern; and when $b+1 \leq i \leq k$, the deterioration phases also replicate their own format. Due to these assumptions, the complexity reduces. By generating equations from (22) to (39) as shown in the appendix, it is straightforward to iteratively express the steady state probabilities in terms of $\pi_{0,0}$, as shown in (40) to (43). By recalling that the sum of all steady state probabilities is equal to 1 , we calculate $\pi_{0,0}$ using (44). Substituting the result back to (40)-(43), closed form solutions of all steady state probabilities could be achieved. By substituting the steady state probabilities into (15) and (16), we can get analytical expressions for the asset availability $A_{s}$ and operation cost $C_{s}$. This method is desirable when $k$ and $m$ are small and predefined.

\section{EXAMPLE}

In this section, the applicability of the model is demonstrated by applying it to the maintenance of a fleet of oil immersed power transformers of different ages. The data presented in this section was obtained through a case study with a major power distribution company in Asia. This case study demonstrates the optimal condition based maintenance strategy for the grid corporation to maintain the continual functionality of power transformers. The cost figures have been converted to UK Pounds using the conversion rates prevalent at the time of the study.

Power transformers have high failure costs, and may cause catastrophic damage on the power network. The time required to maintain a transformer is non-negligible, and during this period the system suffers a service reliability drop. Therefore, the availability of power transformers is normally deemed as the bottleneck of reliability and capability in power transmission systems [41], [42]. Hence, its maintenance has been of considerable interest for the reliability management community.

Power transformers consist of critical components such as winding insulation paper; and non-critical components such as cooling equipment, bushing, and tank. The life of a power transformer is primarily constrained by its winding insulation paper with an expected service life of 38 years [43]. The condition of winding insulation is normally quantified by the degree of polymerization [44], and assessed through Furan analysis [45]. In CIGRE [4], the condition is classified into five condition states: normal, aged, defective, faulty, and failed. We denote the first 4 deterioration states as $i=0, i=1, i=2$, and $i=3$; and denote the failure state as $F$. The winding paper insulation is subject to two types of accelerated deteriorations. Firstly, it is subject to accelerated chemical degradation, which is caused by aggressive pyrolysis, hydrolysis, and oxidation. The degradation happens in the presence of decay agents such as water, oxygen, acid, and sludge. The excessive decay agents could be attributed to the malfunctioning of non-critical components, such as poor tank sealing, and inelastic gaskets in bushings. Under this circumstance, it could accelerate the aging to as much as 20 times [25]. Mechanical deterioration may be accelerated by an increase in the level of vibration, which is caused by malfunctions such as the loosening of clamps, or the distortion of the geometry of its winding. The lifetime of the winding insulation is approximately reduced to 8 months, under accelerated mechanical deterioration. Rates of the chemical, and mechanical accelerated degradation are 0.001 , and 0.003 per year respectively. The symptoms of accelerated deterioration can be detected by inspection, and remedy actions such as oil reclaiming, drying, de-sludging, reclamping, and repacking are performed to rectify accelerated deterioration. Power transformers also could stop functioning due to external incidents such as short circuits, switch transients, and lightning strikes. In the normal condition, the probability of such a sudden failure is 0.008 per year. Under the presence of decay agents or a reduction of clamping force, the dielectric and mechanical withstand strength are reduced; therefore, the failure probability increases to 0.048 per year.

The deterioration of a power transformer is not revealed, but the failed states are self-announcing. We consider two types of maintenance regimes: corrective maintenance after sudden failure, and condition based maintenance to preventively 
maintain the power transformer based on the inspected condition.

1. To resolve the deterioration failure, a replacement process can last 120 days, which includes cleanup, shipping, and installing. It has a $£ 1 \mathrm{M}$ penalty cost associated with it.

2. If the power transformer suddenly fails, corrective maintenance will be arranged immediately. The statistical mean duration of corrective maintenance is 30 days, with an average cost of $£ 5600$. The corrective maintenance restores the condition of the power transformer to its pervious operation state just before the failure.

3. The major preventive maintenance is able to eliminate the accumulated deterioration, and restore the transformer to an as good as new state through this action. It may involve preventively replacing some of the components. The duration of the major preventive maintenance is 15 days, and costs $£ 600 \mathrm{k}$ on average.

4. If a power transformer undergoes accelerated deterioration, a minor preventive maintenance will be scheduled to repair the malfunctioned non-critical component with an average duration of 4 days, and an average cost of $£ 1900$.

Moreover, an additional planned downtime cost of $£ 3200$ per day is associated with scheduled preventive maintenance actions. However, the penalty of unplanned downtime, which is attributed to sudden failure and deterioration failure, is $£ 53000$ per day. We aim to explore maintenance strategies and inspection strategies to maximize the availability and minimise the cost, by adjusting the major preventive maintenance threshold $(b)$, and MTBI $\left(1 / \lambda_{\text {in }}\right)$. For simplicity, we assume that the failure probabilities and deterioration rates are homogenous, and the sojourn time at each state is governed by an exponential clock. The parameter settings are summarised in Table I.

Table I

Parameter setting of power transformer maintenance

\begin{tabular}{llll}
\hline \hline Parameter & Value & Parameter & Value \\
\hline$\lambda_{n}$ & $0.105 /$ year & $\mu_{M}$ & 24.39 / year \\
$\lambda_{d 1}$ & 2.105 / year & $\mu_{R}$ & $3.04 /$ year \\
$\lambda_{d 2}$ & 5.333 / year & $C_{u}$ & $£ 53000 /$ day \\
$\lambda_{f 1}$ & 0.001 year & $C_{p}$ & $£ 3200 /$ day \\
$\lambda_{f 2}$ & $0.003 /$ year & $C_{M}^{\prime}$ & $£ 600000$ \\
$\lambda_{F}$ & $0.008 /$ year & $C_{F}^{\prime}$ & $£ 5600$ \\
$\lambda_{F d}$ & $0.048 /$ year & $C_{c}^{\prime}$ & $£ 1900$ \\
$\mu_{i n}$ & $1095 /$ year & $C_{R}^{\prime}$ & $£ 1000000$ \\
$\mu_{c}$ & $91.25 /$ year & $C_{i n}^{\prime}$ & $£ 1000$ \\
$\mu_{F}$ & $12.05 /$ year & & \\
\hline \hline
\end{tabular}

This power transformer deterioration and maintenance can be modelled as illustrated in Fig. 5.

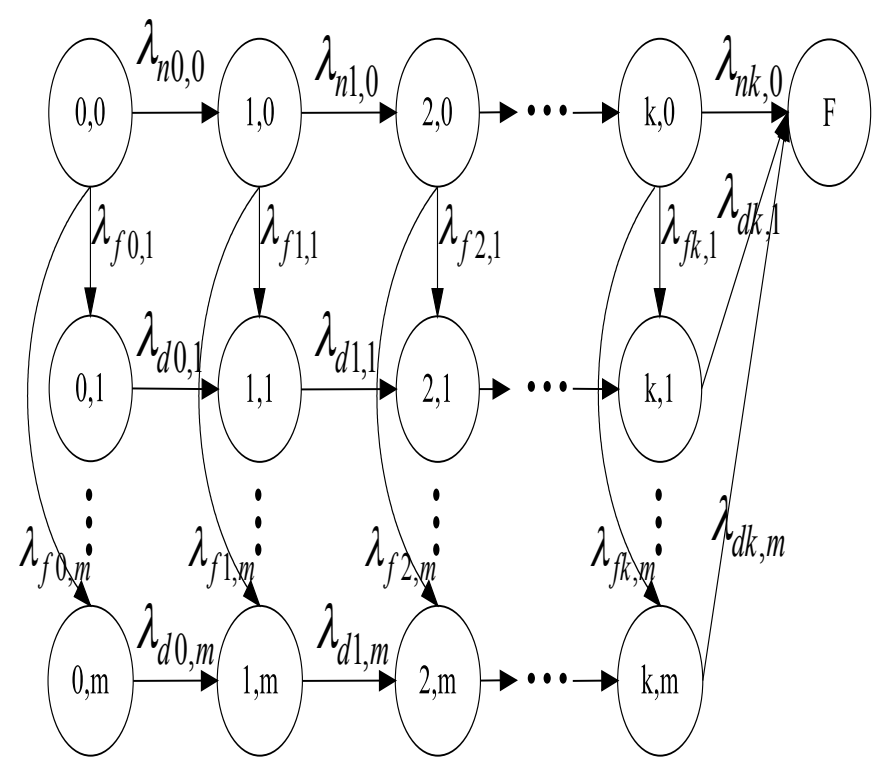

Fig. 5. State transition diagram of power transformer maintenance.

Because power transformers can exist in one of five states, we only need to consider three candidate preventive maintenance strategies: major preventive maintenance at aged state $(b=0)$, at defective state $(b=1)$, and at faulty state $(b=2)$. Using the method described in Section IV, the availability and cost at different MTBI for all major maintenance thresholds can be computed. The results are plotted in Figs. 6 and 7.

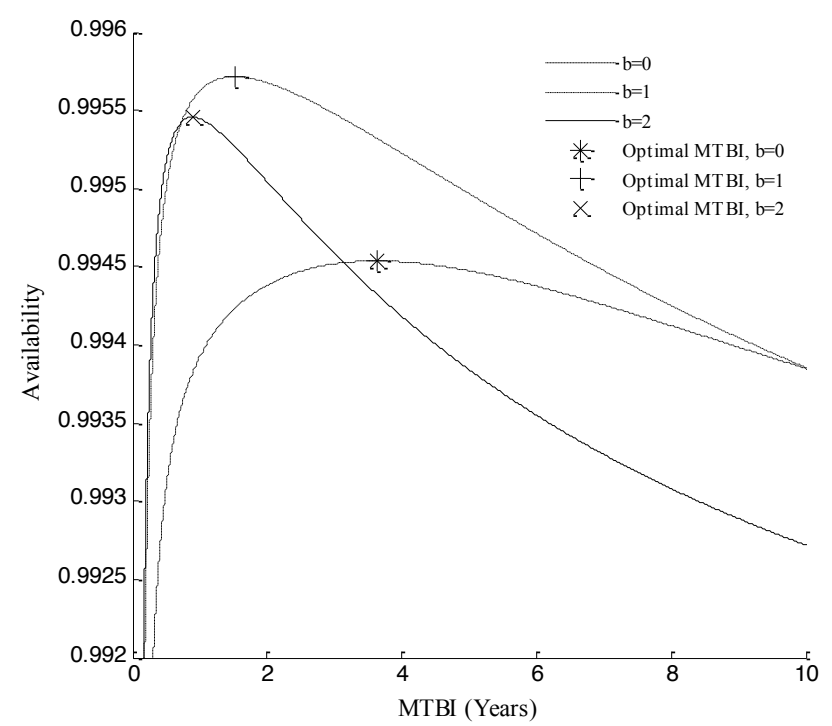

Fig. 6. Availability oriented inspection and maintenance strategies. 


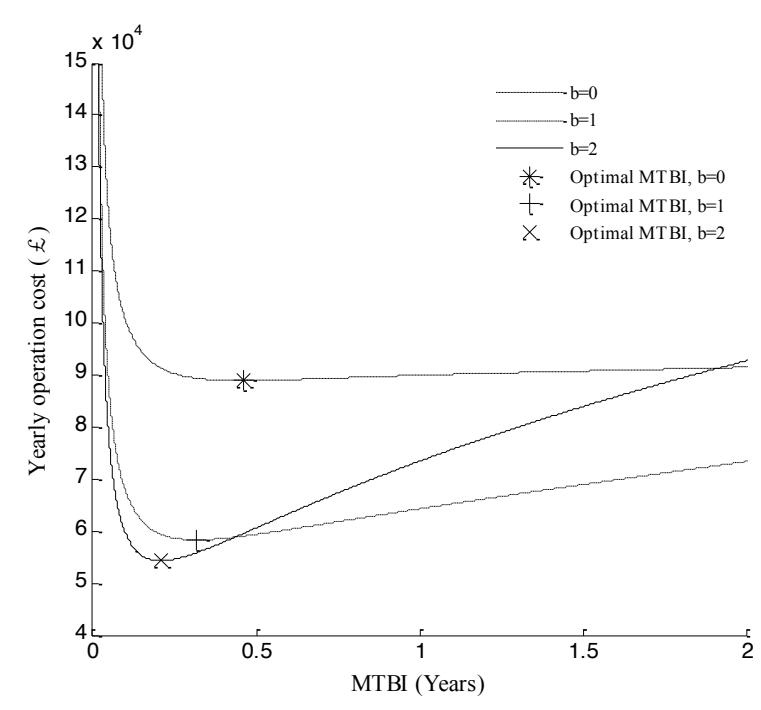

Fig. 7. Cost oriented inspection and maintenance strategies.

The optimal MTBI to maximize availability $\left(1 / \lambda_{\text {oa }}\right)$ could be found by solving (19).

$\frac{d A_{S}}{d \lambda_{\text {in }}}=0$

The optimal MTBI to minimize cost $\left(1 / \lambda_{o c}\right)$ can be found by solving (20).

$\frac{d C_{S}}{d \lambda_{\text {in }}}=0$

We denote the availability at $\lambda_{o a}$ as $A_{o}$, and cost at $\lambda_{o c}$ as $C_{o}$. Tables II and III present $\lambda_{o a}, \lambda_{o c}, A_{o}$, and $C_{o}$ under different values of major maintenance thresholds (b).

Table II

Optimal availability oriented inspection strategies

\begin{tabular}{lll}
\hline \hline & $\begin{array}{l}\text { Optimal availability oriented } \\
\text { MTBI }\left(\mathbf{1} / \boldsymbol{\lambda}_{\boldsymbol{o}}\right)\end{array}$ & $\begin{array}{l}\text { Optimal availability } \\
\left(\boldsymbol{A}_{\boldsymbol{o}}\right)\end{array}$ \\
\hline $\mathrm{b}=0$ & 3.636 years & 0.9945 \\
$\mathrm{~b}=1$ & 1.526 years & 0.9957 \\
$\mathrm{~b}=2$ & 0.898 years & 0.9955 \\
\hline \hline
\end{tabular}

Table III

Optimal cost oriented inspection strategies

\begin{tabular}{lll}
\hline \hline & $\begin{array}{l}\text { Optimal cost oriented MTBI } \\
\left(\mathbf{1} / \boldsymbol{\lambda}_{\boldsymbol{o c}}\right)\end{array}$ & $\begin{array}{l}\text { Minimum yearly } \\
\text { operation cost }\left(\boldsymbol{C}_{\boldsymbol{o}}\right)\end{array}$ \\
\hline $\mathrm{b}=0$ & 0.459 years & $£ 88919$ \\
$\mathrm{~b}=1$ & 0.319 years & $£ 58312$ \\
$\mathrm{~b}=2$ & 0.209 years & $£ 54296$ \\
\hline \hline
\end{tabular}

From Table II, we can see that the maximum availability is $99.57 \%$, which could be attained when the asset is inspected every 1.526 years with major preventive maintenance at encountering a defective state. Table III implies that, with a 0.209 years MTBI, and major preventive maintenance at encountering a faulty state, the asset operational cost is minimized to $£ 54296 /$ year. Figs. 6 and 7 indicate that, when $b=1$, both availability and cost are less sensitive to the change of MTBI when compared $b=2$.

We could set $\lambda_{f 1}$ and $\lambda_{f 2}$ to zero to imitate the scenario without accelerated deterioration. Through the same calculation, the $\lambda_{o a}$ and $\lambda_{o c}$ can be computed, and the results are presented in Table IV.

Table IV

Optimal availability oriented inspection strategies for maintenance strategies with assumption of zero accelerated deterioration

\begin{tabular}{lll}
\hline \hline & $\begin{array}{l}\text { Optimal availability oriented } \\
\text { MTBI }\left(\mathbf{1} / \boldsymbol{\lambda}_{\text {ou }}\right)\end{array}$ & $\begin{array}{l}\text { Optimal cost oriented } \\
\text { MTBI }\left(\mathbf{1} / \boldsymbol{\lambda}_{\boldsymbol{~}}\right)\end{array}$ \\
\hline $\mathrm{b}=0$ & 4.125 year & 2.6102 year \\
$\mathrm{b}=1$ & 1.9687 year & 0.8145 year \\
$\mathrm{b}=2$ & 1.098 year & 0.3029 year \\
\hline \hline
\end{tabular}

These optimal strategies with the assumption of no accelerated deterioration however will deviate from the optimal under the presence of accelerated deterioration. The improvement on availability and cost can be deemed as the benefit of modelling accelerated deterioration, as demonstrated in Table V.

Table V

Improvement on availability cost with accelerated deterioration

\begin{tabular}{lll}
\hline \hline & $\begin{array}{l}\text { Improvement on optimal } \\
\text { availability }\left(\boldsymbol{A}_{\boldsymbol{o}}\right)\end{array}$ & $\begin{array}{l}\text { Reduction on optimal } \\
\text { yearly operation cost }\left(\boldsymbol{C}_{\boldsymbol{o}}\right)\end{array}$ \\
\hline $\mathrm{b}=0$ & $9.1 \times 10^{-6}$ & $£ 3731$ \\
$\mathrm{~b}=1$ & $3.24 \times 10^{-5}$ & $£ 4109$ \\
$\mathrm{~b}=2$ & $2.87 \times 10^{-5}$ & $£ 1178$ \\
\hline
\end{tabular}

In Table $\mathrm{V}$, a negligible improvement on availability is achieved by considering accelerated deterioration. This improvement is mainly because the power transformer has a high reliability, and malfunction rates $\left(\lambda_{f 1}\right.$ and $\left.\lambda_{f 2}\right)$ are very small. However, due to the high failure cost and down time penalty, the savings from operations costs is significant. It can save as much as $7 \%$ of yearly operations costs.

A finding from Fig. 6 and 7 is that major preventive maintenance at defective state $b(=1)$ has the best potential to minimize the unavailability, while major preventive maintenance at a faulty state $(b=2)$ is the best method for saving on yearly operations costs. To further investigate the overall performance with considering both unavailability $\left(1-A_{s}\right)$ and operations costs, we then move our analysis from a single objective optimization to a multi-objective optimization. Fig. 8 plots Pareto fronts for inspection strategies with three different major maintenance threshold settings. 


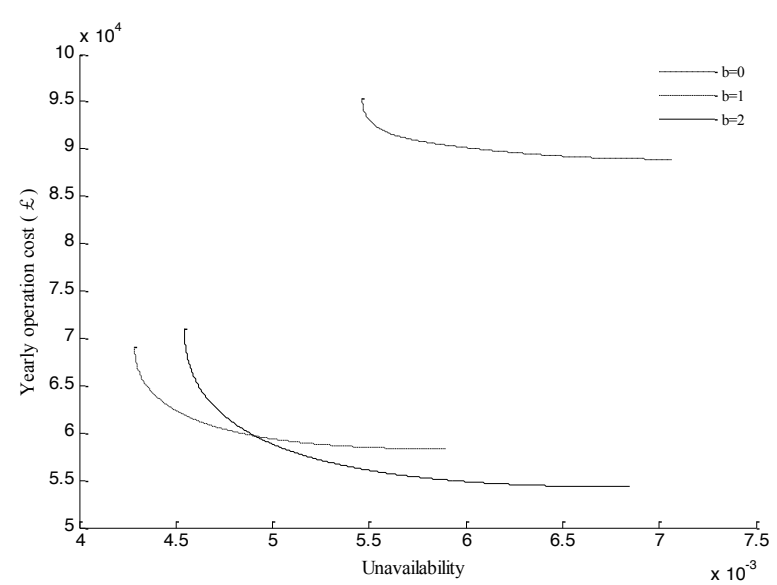

Fig. 8. Pareto fronts when $b=1, b=2$, and $b=3$.

A trade-off between yearly operation cost and unavailability exists in all values of $b$. We can see that the performance when $b=1$, and $b=2$ dominates the performance when $b=0$. We can also see that the Pareto fronts of $b=1$ and $b=2$ cross at $\left(U_{t h}, C_{t h}\right)$. These results suggest that, if the target unavailability $\left(U_{r}\right)$ is lower than $U_{t h}$, the maintenance strategy with $b=1$ has better performance. If the budget of yearly operations $\operatorname{cost}\left(C_{r}\right)$ is below $C_{t h}$, maintenance strategy with $b=2$ is preferred. In this case, $U_{t h}=0.0049$, and $C_{t h}=£ 5967$. Preferred zones of $b=1$ and $b=2$ could be classified as (21).

$$
b=\left\{\begin{array}{cc}
1 & U_{r}<0.0049, C_{r}>£ 59670 \\
1 \text { or } 2 & U_{r} \geq 0.0049 C_{r} \geq £ 59670 \\
2 & U_{r}>0.0049 C_{r}<£ 59670 \\
\varnothing & U_{r}<0.0049 C_{r}<£ 59670
\end{array}\right.
$$

The result aligns with intuition. By postponing the major preventive maintenance threshold, the operations cost of the power transformer is reduced. If availability is important and needs to be increased, the solution is to perform maintenance earlier. - However, this approach could be inefficient due to the non-negligible maintenance duration.

\section{V.CONCLUSION}

In this paper, we presented a novel modelling technique for the deterioration and maintenance of industrial assets with accelerated deterioration associated with fault propagation. We showed that the resulting accelerated deterioration caused by fault propagation can have a non-negligible impact on asset deterioration. A condition based maintenance model is designed, which exhibits a good mathematical tractability. Iterative closed form expressions of availability and cost are developed. We also demonstrated that condition based maintenance with minor preventive maintenance to rectify the accelerated deterioration may achieve a better performance than the one neglecting accelerated deterioration. Further research is suggested in the direction of converting the Markov process to a semi-Markov process model by relaxing the assumption of exponential sojourn times.

\section{APPENDIX: Closed Form SOLUTIONS FOR THE GENERALIZED CONTINUOUS TIME MARKOV CHAIN MAINTENANCE MODEL}

Equilibrium equations at each state in Fig. 4 are shown from (22) to (39).

At state $(0,0)$,

$\pi_{0, m+4} \mu_{F}+\sum_{i=b+1}^{k} \pi_{i, m+3} \mu_{M}+\pi_{F} \mu_{R}+\pi_{0, m+1} \mu_{i n}-\left(\lambda_{F}+\lambda_{n 0,0}+\sum_{j=1}^{m} \lambda_{f 0, j}+\lambda_{i n}\right) \pi_{0,0}=\frac{\partial \pi_{0,0}}{\partial t}$.

At state $(0, j)$ when $1 \leq j \leq m$,

$\pi_{0, j} \lambda_{f 0, j}-\left(\lambda_{F d}+\lambda_{d 0, j}+\lambda_{i n}\right) \pi_{0, j}=\frac{\partial \pi_{0, j}}{\partial t}$.

(23)

At state $(0, m+1)$,

$\pi_{0,0} \lambda_{\text {in }}-\pi_{0, m+1} \mu_{\text {in }}=\frac{\partial \pi_{0, m+1}}{\partial t}$.

At state $(0, m+2)$,

$\lambda_{i n} \sum_{j=1}^{m} \pi_{0, j}-\pi_{0, m+2} \mu_{i n}=\frac{\partial \pi_{0, m+2}}{\partial t}$.

At state $(0, m+3)$,

$\pi_{0, m+2} \mu_{i n}-\pi_{0, m+3} \mu_{c}=\frac{\partial \pi_{0, m+3}}{\partial t}$.

(26)

At state $(0, m+4)$,

$\lambda_{F d} \sum_{j=1}^{m} \pi_{0, j}+\lambda_{F} \pi_{0,0}-\mu_{F} \pi_{0, m+4}=\frac{\partial \pi_{0, m+4}}{\partial t}$.

At state $(i, 0)$ when $1 \leq i \leq \mathrm{b}$,

$\pi_{i-1,0} \lambda_{n i-1,0}+\pi_{i, m+4} \mu_{F}+\pi_{i, m+3} \mu_{c}+\pi_{i, m+1} \mu_{i n}-\left(\lambda_{F}+\lambda_{n i, 0}+\sum_{j=1}^{m} \lambda_{f i, j}+\lambda_{i n}\right) \pi_{i, 0}=\frac{\partial \pi_{i, 0}}{\partial t}$. 
At state $(i, j)$ when $1 \leq i \leq \mathrm{b}$ and $1 \leq j \leq m$,

$\pi_{i-1, j} \lambda_{d i-1, j}+\pi_{i, 0} \lambda_{f i, j}-\left(\lambda_{d i, j}+\lambda_{F d}+\lambda_{i n}\right) \pi_{i, j}=\frac{\partial \pi_{i, j}}{\partial t}$.

At state $(i, m+1)$ when $1 \leq i \leq \mathrm{b}$,

$\pi_{i, 0} \lambda_{\text {in }}-\pi_{i, m+1} \mu_{\text {in }}=\frac{\partial \pi_{i, m+1}}{\partial t}$.

At state $(i, m+2)$ when $1 \leq i \leq \mathrm{b}$,

$\lambda_{i n} \sum_{j=1}^{m} \pi_{i, j}-\pi_{i, m+2} \mu_{i n}=\frac{\partial \pi_{i, m+2}}{\partial t}$.

At state $(i, m+3)$ when $1 \leq i \leq \mathrm{b}$,

$\pi_{i, m+2} \mu_{i n}-\pi_{i, m+3} \mu_{c}=\frac{\partial \pi_{i, m+3}}{\partial t}$.

At state $(i, m+4)$ when $1 \leq i \leq \mathrm{b}$,

$\lambda_{F} \pi_{i, 0}+\lambda_{F d} \sum_{j=1}^{m} \pi_{i, j}-\pi_{i, m+4} \mu_{F}=\frac{\partial \pi_{i, m+4}}{\partial t}$.

At state $(i, 0)$ when $\mathrm{b} \leq i \leq \mathrm{k}$,

$\pi_{i-1,0} \lambda_{n i-1,0}+\pi_{i, m+4} \mu_{F}-\left(\lambda_{F}+\lambda_{n i, 0}+\sum_{j=1}^{m} \lambda_{f i, j}+\lambda_{i n}\right) \pi_{i, 0}=\frac{\partial \pi_{i, 0}}{\partial t}$.

At state $(i, j)$ when $\mathrm{b} \leq i \leq \mathrm{k}$ and $1 \leq j \leq m$,

$\pi_{i-1, j} \lambda_{d i-1, j}+\pi_{i, 0} \lambda_{f i, j}-\left(\lambda_{d i, j}+\lambda_{i n}+\lambda_{F d}\right) \pi_{i, j}=\frac{\partial \pi_{i, j}}{\partial t}$.

At state $(i, m+2)$ when $\mathrm{b} \leq i \leq \mathrm{k}$,

$\lambda_{i n} \sum_{j=0}^{m} \pi_{i, j}-\mu_{i n} \pi_{i, m+2}=\frac{\partial \pi_{i, m+2}}{\partial t}$.

At state $(i, m+3)$ when $\mathrm{b} \leq i \leq \mathrm{k}$,

$\mu_{i n} \pi_{i, m+2}-\mu_{m} \pi_{i, m+3}=\frac{\partial \pi_{i, m+3}}{\partial t}$.

(37)

At state $(i, m+4)$ when $\mathrm{b} \leq i \leq \mathrm{k}$,

$\lambda_{F} \pi_{i, 0}+\lambda_{F d} \sum_{j=1}^{m} \pi_{i, j}-\mu_{F} \pi_{i, m+4}=\frac{\partial \pi_{i, m+4}}{\partial t}$.

At state $\mathrm{F}$,

$\lambda_{n k, 0} \pi_{k, 0}+\sum_{j=1}^{m} \lambda_{d, j} \pi_{k, j}-\mu_{R} \pi_{F}=\frac{\partial \pi_{F}}{\partial t}$.

We can analytically represent the expression of each state in terms of $\pi_{0,0}$, as demonstrated in (40) through (43).

$$
\pi_{0, j}=\left\{\begin{array}{r}
\pi_{0,0}, j=0 \\
\frac{\lambda_{f 0, j} \pi_{0,0}}{\lambda_{F d}+\lambda_{d 0, j}+\lambda_{i n}}, 1 \leq j \leq m \\
\frac{\lambda_{i n} \pi_{0,0}}{\mu_{i n}}, j=m+1 \\
\frac{\lambda_{i n} \sum_{l=1}^{m} \pi_{0, l}}{\mu_{i n}}, j=m+2 \\
\frac{\lambda_{i n} \sum_{l=1}^{m} \pi_{0, l}}{\mu_{c}}, j=m+3 \\
\frac{\lambda_{f 0, l} \pi_{0,0}}{\lambda_{F d} \pi_{d 0, l}+\lambda_{i n}}, j=m+4
\end{array}\right.
$$




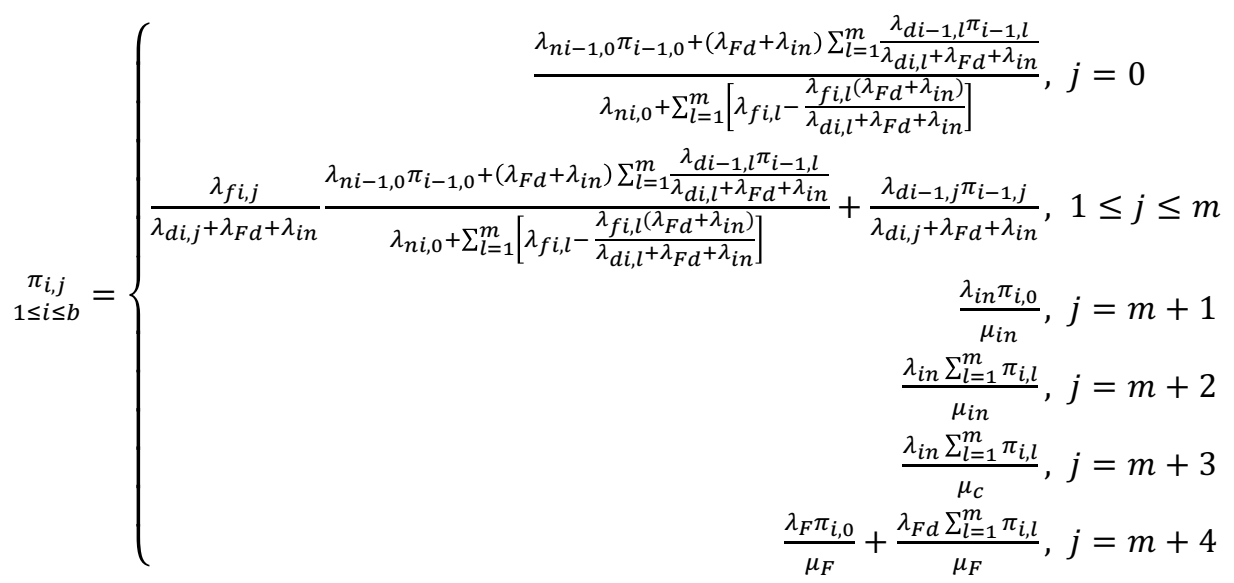

$$
\begin{aligned}
& \int \frac{\lambda_{n i-1,0} \pi_{i-1,0}+\lambda_{F d} \sum_{l=1}^{m} \frac{\lambda_{d i-1, l} \pi_{i-1, l}}{\lambda_{d i, l}+\lambda_{F d}+\lambda_{i n}}}{\lambda_{n i, 0}+\lambda_{i n}+\sum_{l=1}^{m}\left(\lambda_{f i, l}-\frac{\lambda_{f i, l} l_{F d}}{\lambda_{d i, l}+\lambda_{F d}+\lambda_{i n}}\right)}, j=0 \\
& \pi_{i, j}=\left\{\begin{array}{r}
\frac{\lambda_{f i, j}}{\lambda_{d i, j}+\lambda_{F d}+\lambda_{i n}} \frac{\lambda_{n i-1,0} \pi_{i-1,0}+\lambda_{F d} \sum_{l=1}^{m} \frac{\lambda_{d i-1, l} \pi_{i-1, l}}{\lambda_{n i, 0}+\lambda_{i n}+\sum_{l=1}^{m}\left(\lambda_{f i, l}-\frac{\lambda_{f i, ~} \lambda_{F d}}{\lambda_{d i, l}+\lambda_{F d}+\lambda_{i n}}\right)}+\frac{\lambda_{d i-1, j} \pi_{i-1, j}}{\lambda_{d i, j}+\lambda_{F d}+\lambda_{i n}},}{N}, j \leq m \\
N . A ., j=m+1 \\
\frac{\lambda_{i n} \sum_{l=0}^{m} \pi_{i, l}}{\mu_{i n}}, j=m+2 \\
\frac{\lambda_{i n} \sum_{l=0}^{m} \pi_{i, l}}{\mu_{M}}, j=m+3 \\
\frac{\lambda_{F} \pi_{i, 0}}{\mu_{F}}+\frac{\lambda_{F d} \sum_{l=1}^{m} \pi_{i, l}}{\mu_{F}}, j=m+4
\end{array}\right.
\end{aligned}
$$

By recalling that the sum of all steady state probabilities is equal to 1 , we have (44).

$\pi_{0,0}=\frac{1}{\sum_{i=0}^{k} \sum_{j=0}^{m+4} \pi_{i, j}+\pi_{F}}$

Therefore, the analytical expression of each state can be calculated by substituting the result from (44) into (40) to (43).

\section{REFERENCES}

[1] S. H. Sim and J. Endrenyi, "A failure-repair model with minimal and major maintenance," Reliability, IEEE Transactions on, vol. 42, no. 1, pp. 134-140, 1993.

[2] G. Pulcini, "On the overhaul effect for repairable mechanical units: a Bayes approach," Reliability Engineering \& System Safety, vol. 70, no. 1, pp. 85-94, 2000.

[3] P. R. Sianipar and T. M. Adams, "Fault-tree model of bridge element deterioration due to interaction," Journal of Infrastructure Systems, vol. 3, no. 3, pp. 103-110, 1997.

[4] B. No, "227 Guidelines for Life Management Techniques for Power Transformers," CIGRE WG, vol. 12.

[5] R. Dekker, R. E. Wildeman, and F. A. van der Duyn Schouten, "A review of multi-component maintenance models with economic dependence," Mathematical
Methods of Operations Research, vol. 45, no. 3, pp. 411-435, 1997.

[6] S. C. Gordon, "Stochastic dependence in competing risks," American Journal of Political Science, pp. 200-217, 2002.

[7] S. Lo and R. A. Wilke, "A copula model for dependent competing risks," Journal of the Royal Statistical Society: Series C (Applied Statistics), vol. 59, no. 2, pp. 359-376, 2010.

[8] Y. Wang and H. Pham, "Modeling the dependent competing risks with multiple degradation processes and random shock using time-varying copulas," Reliability, IEEE Transactions on, vol. 61, no. 1, pp. 13-22, 2012.

[9] X.-S. Tang, D.-Q. Li, C.-B. Zhou, K.-K. Phoon, and L.-M. Zhang, "Impact of copulas for modeling bivariate distributions on system reliability," Structural Safety, vol. 44, pp. 80-90, 2013.

[10] L. Bian and N. Gebraeel, "Stochastic Modeling and Real-Time Prognostics for Multi-Component Systems 
with Degradation-Rate-Interactions," IIE Transactions, no. just-accepted, 2013.

[11] R. P. Nicolai and R. Dekker, Optimal maintenance of multi-component systems: a review. Springer, 2008.

[12] D. N. P. Murthy and D. G. Nguyen, "Study of two-component system with failure interaction," Naval Research Logistics Quarterly, vol. 32, no. 2, pp. 239-247, 1985.

[13] P. A. Scarf and M. Deara, "Block replacement policies for a two-component system with failure dependence," Naval Research Logistics (NRL), vol. 50, no. 1, pp. 70-87, 2003.

[14] M.-T. Lai, "A discrete replacement model for a two-unit parallel system subject to failure rate interaction," Quality and Quantity, vol. 43, no. 3, pp. 471-479, 2009.

[15] H. R. Golmakani and H. Moakedi, "Periodic inspection optimization model for a two-component repairable system with failure interaction," Computers \& Industrial Engineering, 2011.

[16] T. Satow and S. Osaki, "Optimal replacement policies for a two-unit system with shock damage interaction," Computers \& Mathematics with Applications, vol. 46, no. 7, pp. 1129-1138, 2003.

[17] G. J. Wang and Y. L. Zhang, "A geometric process repair model for a two-component system with shock damage interaction," International Journal of Systems Science, vol. 40, no. 11, pp. 1207-1215, 2009.

[18] I. A. Watson and G. T. Edwards, "Common-mode failures in redundancy systems," Nucl. Technol.;(United States), vol. 46, no. 2, 1979.

[19] A. Mosleh, "Common cause failures: an analysis methodology and examples," Reliability Engineering \& System Safety, vol. 34, no. 3, pp. 249-292, 1991.

[20] P. H. Kvam and H. F. Martz, "Bayesian inference in a discrete shock model using confounded common cause data," Reliability Engineering \& System Safety, vol. 48, no. 1, pp. 19-25, 1995.

[21] L. Xing and G. Levitin, "Combinatorial analysis of systems with competing failures subject to failure isolation and propagation effects," Reliability Engineering \& System Safety, vol. 95, no. 11, pp. 1210-1215, 2010.

[22] G. Levitin, "Incorporating common-cause failures into nonrepairable multistate series-parallel system analysis," Reliability, IEEE Transactions on, vol. 50, no. 4, pp. 380-388, 2001.

[23] G. Levitin and L. Xing, "Reliability and performance of multi-state systems with propagated failures having selective effect," Reliability Engineering \& System Safety, vol. 95, no. 6, pp. 655-661, 2010.

[24] G. Levitin, L. Xing, H. Ben-Haim, and Y. Dai, "Reliability of Series-Parallel Systems With Random
Failure Propagation Time," 2013.

[25] D. H. Shroff and A. W. Stannett, "A review of paper aging in power transformers," Generation, Transmission and Distribution, IEE Proceedings $C$, vol. 132, no. 6, pp. $312-319$, Nov. 1985.

[26] S. H. Sim and J. Endrenyi, "Optimal preventive maintenance with repair," Reliability, IEEE Transactions on, vol. 37, no. 1, pp. 92-96, 1988.

[27] H. Pham, A. Suprasad, and R. B. Misra, "Availability and mean life time prediction of multistage degraded system with partial repairs," Reliability Engineering \& System Safety, vol. 56, no. 2, pp. 169-173, 1997.

[28] S. V. Amari and L. McLaughlin, "Optimal design of a condition-based maintenance model," Reliability and Maintainability, 2004 Annual Symposium-RAMS, 2004, pp. 528-533.

[29] D. Chen and K. S. Trivedi, "Closed-form analytical results for condition-based maintenance," Reliability Engineering \& System Safety, vol. 76, no. 1, pp. 43-51, 2002.

[30] D. Chen, Y. Cao, K. S. Trivedi, and Y. Hong, "Preventive maintenance of multi-state system with phase-type failure time distribution and non-zero inspection time," International journal of reliability, quality and safety engineering, vol. 10, no. 03, pp. 323-344, 2003.

[31] P. Rao and V. N. Naikan, "An Optimization Methodology for Condition Based Minimal and Major Preventive Maintenance," Economic Quality Control, vol. 21, no. 1, pp. 127-141, 2006.

[32] S. Ambani, L. Li, and J. Ni, "Condition-based maintenance decision-making for multiple machine systems," Journal of Manufacturing Science and Engineering, vol. 131, no. 3, p. 31009, 2009.

[33] I. W. Soro, M. Nourelfath, and D. Aït-Kadi, "Performance evaluation of multi-state degraded systems with minimal repairs and imperfect preventive maintenance," Reliability Engineering \& System Safety, vol. 95, no. 2, pp. 65-69, 2010.

[34] R. Agepati, N. Gundala, and S. V. Amari, "Optimal software rejuvenation policies," Reliability and Maintainability Symposium (RAMS), 2013 Proceedings-Annual, 2013, pp. 1-7.

[35] W. Li and H. Pham, "Reliability modeling of multi-state degraded systems with multi-competing failures and random shocks," Reliability, IEEE Transactions on, vol. 54, no. 2, pp. 297-303, 2005.

[36] S. V. Amari, L. McLaughlin, and H. Pham, "Cost-effective condition-based maintenance using markov decision processes," Reliability and Maintainability Symposium, 2006. RAMS '06. Annual, 2006, pp. 464-469. 
[37] D. Montoro-Cazorla and R. Pérez-Ocón, “A deteriorating two-system with two repair modes and sojourn times phase-type distributed," Reliability Engineering \& System Safety, vol. 91, no. 1, pp. 1-9, 2006.

[38] M. F. Neuts, "Probability distributions of phase type," Liber Amicorum Prof. Emeritus H. Florin, vol. 173, p. 206, 1975.

[39] T. M. Welte, "A rule-based approach for establishing states in a Markov process applied to maintenance modelling," Proceedings of the Institution of Mechanical Engineers, Part O: Journal of Risk and Reliability, vol. 223, no. 1, pp. 1-12, 2009.

[40] W. Kuo and M. J. Zuo, Optimal reliability modeling: principles and applications. Wiley, 2002.

[41] A. van Schijndel, "Power transformer reliability modelling," Dissertation, Eindhoven University of Technology, 2010.

[42] A. E. Abu-Elanien and M. M. A. Salama, "Asset management techniques for transformers," Electric power systems research, vol. 80, no. 4, pp. 456-464, 2010.

[43] D. H. Shroff and A. W. Stannett, "A review of paper aging in power transformers," IEE Proceedings C (Generation, Transmission and Distribution), 1985, vol. 132, pp. 312-319.

[44] A. M. Emsley and G. C. Stevens, "Review of chemical indicators of degradation of cellulosic electrical paper insulation in oil-filled transformers," IEE Proceedings-Science, Measurement and Technology, vol. 141, no. 5, pp. 324-334, 1994.

[45] L. Cheim, D. Platts, T. Prevost, and S. Xu, "Furan analysis for liquid power transformers," Electrical Insulation Magazine, IEEE, vol. 28, no. 2, pp. 8-21, 2012.

Zhenglin Liang is a Ph.D. student at the Institute for Manufacturing, University of Cambridge. Zhenglin completed a BEng degree with $1^{\text {st }}$ class honors in electrical and electronic engineering at Manchester University, and a MEng degree in communication and signal processing at Bristol University. His research interests are in condition based maintenance, stochastic dependence, and power transformer maintenance.

Ajith Kumar Parlikad received the Ph.D. degree in manufacturing management from Cambridge University, Cambridge, U.K., in 2006. He is now a Senior Lecturer in Industrial Systems with Cambridge University, Cambridge, U.K. His research interests include asset investment and maintenance decision-making, particularly based on value. His research has been funded by the EPSRC and industry. He sits on the Executive Committee in the Institution of Engineering and Technology TPN on Asset Management, U.K., and is the Chair of the Academic and Research Network for the Institute of Asset Management, U.K. 